\section{Case Reports in Ophthalmology}

Case Rep Ophthalmol 2019;10:32-40

DOI: $10.1159 / 000496384$

Published online: January 24, 2019 (c) 2019 The Author(s)

Published by S. Karger AG, Basel www.karger.com/cop

This article is licensed under the Creative Commons Attribution-NonCommercial 4.0 International License (CC BY-NC) (http://www.karger.com/Services/OpenAccessLicense). Usage and distribution for commercial purposes requires written permission.

\title{
Neurosarcoidosis Occurring 6 Years after Onset of Vogt-Koyanagi-Harada Disease
}

\author{
Yoshihide Sehara ${ }^{a}$ b Hideaki Otsuka ${ }^{a}$ Shinichi Sakamotoc, d \\ Yoshihito Ando ${ }^{a}$ Mikio Sawada ${ }^{a}$ \\ aDepartment of Neurology, Haga Red Cross Hospital, Moka, Japan; bDivision of Genetic \\ Therapeutics, Center for Molecular Medicine, Jichi Medical University, Shimotsuke, Japan; \\ 'Department of Ophthalmology, Haga Red Cross Hospital, Moka, Japan; dDepartment of \\ Ophthalmology, Jichi Medical University, Shimotsuke, Japan
}

\section{Keywords}

Neurosarcoidosis $\cdot$ Steroid therapy $\cdot$ Vogt-Koyanagi-Harada disease

\begin{abstract}
A 43-year-old Japanese woman visited a clinic with a 2-week history of visual loss, hyperemia, and bilateral eye pain. Dilated fundus and optical coherence tomography showed hyperemic optic disc and multifocal serous retinal detachments. Fundus fluorescein angiography revealed optic disc leakage and numerous hyperfluorescent pinpoints of leakage, which are typical of Vogt-Koyanagi-Harada disease (VKHD). She also showed tinnitus, poliosis, and alopecia. With a diagnosis of VKHD, steroid therapy was started. At the age of 48, oral prednisolone was tapered off, and only corticosteroid eye drops were continued. Eighteen months later, dysesthesia appeared in the left side of her face and in her left ring and little fingers, which spread to the ulnar side of her forearm. Blood tests indicated elevated serum angiotensin-converting enzyme (ACE) levels. Chest CT showed bilateral lymphadenopathy, and histological analysis of the subcutaneous mass in her right arm showed multinucleated giant cells. Steroid therapy was started under a diagnosis of sarcoidosis. Serum ACE and lysozyme levels decreased in response to steroid therapy. We report a case of the concurrence of VKHD and sarcoidosis, which may share a common pathophysiology. Accumulation of further similar cases is necessary to elucidate the precise mechanism underlying the concurrence of these two diseases.
\end{abstract}




\section{Introduction}

Sarcoidosis is a multiorgan granulomatous inflammatory disease of unknown etiology, which is characterized by the formation of multiple noncaseating granulomas [1]. The incidence rate shows variability throughout the world with a difference in racial and geographical distribution. In Japan, the annual incidence is 1 per 100,000 [2]. Although sarcoidosis most often affects the lungs and intrathoracic lymph nodes, no organs or tissues are spared from sarcoidosis, and it has been reported to involve the eyes, skin, heart, gastrointestinal tract, liver, and joints [3]. Neurosarcoidosis, the involvement of the central and peripheral nervous system by sarcoid granulomata, is uncommon, and diagnosis can be challenging if not clinically suspected. Neurosarcoidosis manifests symptomatically in $5 \%$ of patients with sarcoidosis [4].

Vogt-Koyanagi-Harada disease (VKHD) is also a rare multisystemic, granulomatous inflammatory condition that targets melanocytic tissues, such as the eye, inner ear, meninges, skin, and hair. VKHD is usually characterized by bilateral panuveitis, which is a common cause of visual disability. The incidence of VKHD varies worldwide. In Japan, the annual incidence of VKHD is 1 per 100,000 [5].

Here, we present a case of a 49-year-old woman with neurosarcoidosis who had been treated for VKHD over a period 6 of years. The incidental concurrence should be rare, and to our knowledge has not been reported previously. As much remains unknown about their etiology, they may share a common pathophysiology.

\section{Case Presentation}

At the age of 43, a Japanese woman visited a clinic with a 2-week history of visual loss, hyperemia, and bilateral eye pain. Her best corrected visual acuity was 0.15 in the right eye and 0.4 in the left eye, and intraocular pressure of $8 \mathrm{~mm} \mathrm{Hg}$ in both eyes. Slit lamp examination showed cells and flare in the anterior chamber of each eye. Dilated fundus and optical coherence tomography showed hyperemic optic disc and multifocal serous retinal detachments. Ultrasonic B-mode examination showed diffuse thickening of the choroid with low to medium reflectivity. Fundus fluorescein angiography revealed optic disc leakage and numerous hyperfluorescent pinpoints of leakage (Fig. 1). She presented with tinnitus, poliosis, and alopecia later on during her clinical course, but not with headache, vertigo, or vitiligo. Serum calcium level was $9.4 \mathrm{mg} / \mathrm{dL}$ (normal rage: $8.8-10.1 \mathrm{mg} / \mathrm{dL}$ ) (angiotensin-converting enzyme [ACE] was not assessed). Cerebrospinal fluid analysis showed that cell count was 11 cells/3 $\mu \mathrm{L}$ (100\% mononuclear) (normal range: $0-5$ cells $/ 3 \mu \mathrm{L}$ ), total protein level was $27 \mathrm{mg} / \mathrm{dL}$ (normal range: $10-40 \mathrm{mg} / \mathrm{dL}$ ), and glucose was $76 \mathrm{mg} / \mathrm{dL}$ (normal range: $50-75 \mathrm{mg} / \mathrm{dL}$ ). Once the diagnosis of VKHD was made, she was treated with methylprednisolone pulse therapy (3 days, $1 \mathrm{~g} /$ day) followed by oral prednisolone (PSL) therapy ( $1 \mathrm{mg} / \mathrm{kg}$ body weight) and corticosteroid eye drops. After commencement of corticosteroid therapy, her best visual acuity recovered temporarily to 1.2 in each eye. However, she gradually lost visual acuity due to cataract development. At the age of 44, she underwent surgeries for phacoemulsification and aspiration with intraocular lens implantation for bilateral cataracts, resulting in recovery of best corrected visual acuity to 1.0 in each eye. At almost the same time, another ocular complication, glaucoma, developed and her visual acuity gradually decreased again. Her fundi showed depigmentation and changed to a reddish glow, which is called "sunset glow fundus" (Fig. 1i, 
j). She became blind in the right eye, and best corrected visual acuity in the left was 0.08 . At the age of 48, oral PSL was tapered off due to the absence of inflammation in each eye.

Her symptoms were stable without oral PSL administration. At the age of 49, she visited a dentist for treatment of her left lower molar. After treatment, dysesthesia like the sensation during local anesthesia remained around her left cheek and spread to the left half of her face. At almost the same time, similar dysesthesia appeared in the left ring and little fingers. One month later, the dysesthesia spread to the ulnar side of her forearm. As the ophthalmological findings were stable, she was referred to our hospital for diagnosis and treatment. She did not have any past medical history except VKHD as described above. She did not have a family history of collagen disease or any other related illness. Her blood pressure was 107/65 mm Hg, heart rate was 76 beats $/ \mathrm{min}$, and body temperature was $36.0^{\circ} \mathrm{C}$. Multiple subcutaneous small tumors were found in her body and extremities. On neurological examination, her right eye was blind, and best corrected visual acuity in the left eye was 0.08 . Dysesthesia and sensory disturbance were observed in the left half of her face, the ulnar side of her forearm, the ulnar side of her left ring finger, and left little finger. Motor, cerebellar, and autonomic examinations yielded no notable findings. Blood test showed a serum calcium level of $9.4 \mathrm{mg} / \mathrm{dL}$ (normal range: 9-11 mg/dL), serum ACE level of $43.7 \mathrm{U} / \mathrm{L}$ (normal range: 8.3-21.4 U/L), serum lysozyme level of $25.4 \mu \mathrm{g} / \mathrm{mL}$ (normal range: $5-10.2 \mu \mathrm{g} / \mathrm{mL}$ ), and serum soluble interleukin-2 receptor level of 4,380 U/mL (normal range: 145-519 U/mL). Anti-DNA antibody, rheumatoid factor, anti-Ro/SSA antibody, anti-La/SSB antibody, anti-thyroglobulin antibody, anti-thyroid peroxidase (TPO) antibody, myeloperoxidase (MPO)-anti-neutrophil cytoplasmic antibody (ANCA), and proteinase 3 (PR3)-ANCA were all negative. Cerebrospinal fluid analysis showed that the cell count was 3 cells $/ 3 \mu \mathrm{L}$ (100\% mononuclear), total protein level was $35 \mathrm{mg} / \mathrm{dL}$, and glucose was $51 \mathrm{mg} / \mathrm{dL}$. Human leukocyte antigen (HLA) findings were B46, B48, DR8, and DR15. Routine nerve conduction study yielded no notable findings. On chest X-ray, it was difficult to identify the abnormality including bilateral hilar lymphadenopathy (BHL). However, chest CT showed enlargement of some mediastinal lymph nodes (Fig. 2a-d). Head and cervical magnetic resonance imaging with contrast yielded no notable findings, including lesions suggestive of inflammation. Histopathological analysis of the subcutaneous tumor in the right arm showed multinucleated giant cells (Fig. 2e). The cause of mononeuropathy multiplex which affected left trigeminal nerve, left ulnar nerve, and left medial cutaneous nerve of forearm was determined to be neurosarcoidosis. One week before admission to our hospital, dysesthesia appeared in the bilateral arms and legs. After admission, she received methylprednisolone pulse therapy ( 3 days, $1 \mathrm{~g} /$ day) followed by oral PSL therapy ( $1 \mathrm{mg} / \mathrm{kg}$ body weight). Although serum ACE and lysozyme levels decreased to $12.4 \mathrm{U} / \mathrm{L}$ and $5.5 \mu \mathrm{g} / \mathrm{mL}, 3$ months after introduction of steroid therapy, dysesthesia and sensory disturbance in the left half of her face and extremities had not changed at the time of this report (Fig. 3).

\section{Discussion}

Here, we reported a case of neurosarcoidosis occurring during 6-year follow-up after diagnosis of VKHD. They may share a common pathophysiology, but the concurrence of these two diseases has not been reported previously.

Although the mechanism underlying VKHD has not been fully elucidated, it is thought to be related to $\mathrm{T}$ cell-mediated autoimmune processes directed against melanocytes. Several studies indicated that $\mathrm{T}$ lymphocytes from patients with VKHD recognized peptides of melanocyte-specific proteins, such as tyrosinase, tyrosinase-related protein 1 and 2 , and 
Pmel17/gp100 [6]. HLA-DR4 is closely associated with VKHD in patients from different ethnic groups, such as American, Chinese, Hispanic, and Japanese patients [7]. Although the HLA in this case did not match, a strong association with HLA-DR4, DRw53, and Bw54 is known, especially among Japanese patients [8]. Moreover, humoral autoantibodies, including antiRo/SSA antibody, are more frequently positive in patients with VKHD than in female blood donors, although the role of anti-Ro/SSA in VKHD has not been elucidated [9]. This case was negative for autoantibodies including anti-Ro/SSA at the time of first visit to our hospital.

As specific markers of VKHD have not yet been found, the diagnosis is primarily based on clinical features. The International Nomenclature Committee proposed comprehensive revised diagnostic criteria for VKHD in 2001 [10]. As this case showed hyperemic optic disc, diffuse thickening of choroid, focal retinal detachments, multiple pinpoint leakage, and optic nerve staining in fluorescein angiography in addition to tinnitus, alopecia, and poliosis, she fulfilled the criteria of complete VKHD. The later clinical feature, such as the reddish glow fundi called "sunset glow fundi" and Dalen-Fuchs atrophic nodules in the retinal periphery also supported the diagnosis of VKHD (Fig.1i-l).

As the clinical presentation of neurosarcoidosis is heterogeneous, diagnosis is difficult if not clinically suspected. In this case, the patient showed dysesthesia on the left side of the face, the ulnar side of the left forearm, and the ring and little fingers of the left hand, which are regions innervated by the left trigeminal nerve, medial antebrachial cutaneous nerve, and ulnar nerve, respectively. As the distribution of affected nerves was that of mononeuropathy multiplex, primary and secondary systemic angiitis, diabetes, and lymphoma were explored for differential diagnoses. In addition to the elevated serum ACE and lysozyme levels and the bilateral hilar lymphadenopathy seen on chest CT scan, histological analysis of the subcutaneous tumor showed multinucleated giant cells, which fulfilled the diagnostic criteria for sarcoidosis [1].

Neurological symptoms are the initial presenting features in approximately $50-70 \%$ of patients with neurosarcoidosis [11]. In a systematic review of 29 articles, $84 \%$ of patients with neurosarcoidosis showed involvement of other organs, including intrathoracic/lungs (57\%), eyes (20\%), skin (18\%), and lymph nodes (18\%). Our case also showed BHL and subcutaneous granulomas at the onset of neurological features. There were no specific ocular findings at the onset of sarcoidosis, possibly because the corticosteroid eye drops had been continued for treatment of VKHD. The most common feature of neurosarcoidosis is cranial neuropathy, in which any cranial nerve may be affected, although cranial nerves II and VII are the most frequently affected $[11,12]$. Peripheral neuropathy occurs in approximately $2-40 \%$ of patients with neurosarcoidosis [12]. Our case showed mononeuropathy multiplex at onset followed by symmetrical polyneuropathy in the period between onset of neurosarcoidosis and admission for steroid therapy.

The etiology of sarcoidosis is unclear. Concerning inherited susceptibility to sarcoidosis, the Case-Control Etiologic Sarcoidosis Study (ACCESS) showed that the likelihood of a first- or second-degree relative with a history of sarcoidosis was fivefold greater for patients than for controls [13]. In addition, it has been established that HLA antigens encoded by HLA-DRB1 and DQB1 alleles are associated with sarcoidosis [14]. As susceptibility to sarcoidosis depends on both genetic and environmental exposure, the interactions between specific sarcoidosis susceptibility loci and environmental modifiers remain unclear [1].

The cardinal pathological feature of granuloma in sarcoidosis is the presence of CD4+ type 1 helper $\mathrm{T}$ lymphocytes and activated macrophages. CD4+ T cells interact with antigen-presenting cells to initiate the formation of granulomas, and activated CD4+ cells differentiate into type 1 helper $\mathrm{T}$ cells, which release cytokines, such as interferon- $\gamma$ and interleukin- 2 , to 
augment macrophage tumor necrosis factor- $\alpha$ production and amplify the local cellular immune response $[1,15]$. Macrophages under chronic cytokine stimulation differentiate into epithelioid cells, lose some phagocytic capacity, and fuse to form multinucleated giant cells.

Although VKHD and sarcoidosis may be relatively common causes of uveitis in referral centers and both are granulomatous inflammatory diseases of unknown etiology, accumulation of information on similar cases is necessary to elucidate the common pathophysiology of these two diseases.

\section{Statement of Ethics}

Written consent was obtained from the patient for publication of this case report.

\section{Disclosure Statement}

The authors declare that they have no conflicts of interest.

\section{Author Contributions}

Y.S. cared for the patient, collected clinical and laboratory data, and drafted and revised the manuscript. H.O. and S.S. cared for the patient, collected clinical and laboratory data, and assisted in drafting the manuscript. Y.A. revised the manuscript. M.S. cared for the patient and revised the manuscript.

\section{References}

1 Iannuzzi MC, Rybicki BA, Teirstein AS. Sarcoidosis. N Engl J Med. 2007 Nov;357(21):2153-65.

2 Pietinalho A, Hiraga Y, Hosoda Y, Löfroos AB, Yamaguchi M, Selroos O. The frequency of sarcoidosis in Finland and Hokkaido, Japan. A comparative epidemiological study. Sarcoidosis. 1995 Mar;12(1):61-7.

3 Tana C, Wegener S, Borys E, Pambuccian S, Tchernev G, Tana M, et al. Challenges in the diagnosis and treatment of neurosarcoidosis. Ann Med. 2015;47(7):576-91.

4 Chen RC, McLeod JG. Neurological complications of sarcoidosis. Clin Exp Neurol. 1989;26:99-112.

5 Iwata D, Mizuuchi K, Aoki K, Horie Y, Kase S, Namba K, et al. Serial Frequencies and Clinical Features of Uveitis in Hokkaido, Japan. Ocul Immunol Inflamm. 2017;25(suppl 1):S15-S18.

6 Damico FM, Cunha-Neto E, Goldberg AC, Iwai LK, Marin ML, Hammer J, et al. T-cell recognition and cytokine profile induced by melanocyte epitopes in patients with HLA-DRB ${ }^{*} 0405$-positive and -negative VogtKoyanagi-Harada uveitis. Invest Ophthalmol Vis Sci. 2005 Jul;46(7):2465-71.

7 Shindo Y, Inoko H, Yamamoto T, Ohno S. HLA-DRB1 typing of Vogt-Koyanagi-Harada's disease by PCR-RFLP and the strong association with DRB1*0405 and DRB1*0410. Br J Ophthalmol. 1994 Mar;78(3):223-6.

8 Kiyomoto C, Imaizumi M, Kimoto K, Abe H, Nakano S, Nakatsuka K. Vogt-Koyanagi-Harada disease in elderly Japanese patients. Int Ophthalmol. 2007 Apr-Jun;27(2-3):149-53.

9 Shinzato M, Yamamoto JH, Hirata CE, Olivalves E, Bonfá E. Anti-SS-A/Ro reactivity in patients with VogtKoyanagi-Harada syndrome. Lupus. 2004;13(4):279-80.

10 Read RW, Holland GN, Rao NA, Tabbara KF, Ohno S, Arellanes-Garcia L, et al. Revised diagnostic criteria for Vogt-Koyanagi-Harada disease: report of an international committee on nomenclature. United States: Am J Ophthalmol; 2001. pp. 647-52.

11 Joseph FG, Scolding NJ. Neurosarcoidosis: a study of 30 new cases. J Neurol Neurosurg Psychiatry. 2009 Mar;80(3):297-304.

12 Hebel R, Dubaniewicz-Wybieralska M, Dubaniewicz A. Overview of neurosarcoidosis: recent advances. J Neurol. 2015 Feb;262(2):258-67. 
Sehara et al.: Neurosarcoidosis Occurring 6 Years after Onset of Vogt-Koyanagi-Harada Disease

13 Rybicki BA, Iannuzzi MC, Frederick MM, Thompson BW, Rossman MD, Bresnitz EA, et al.; ACCESS Research Group. Familial aggregation of sarcoidosis. A case-control etiologic study of sarcoidosis (ACCESS). Am J Respir Crit Care Med. 2001 Dec;164(11):2085-91.

14 Sato H, Woodhead FA, Ahmad T, Grutters JC, Spagnolo P, van den Bosch JM, et al. Sarcoidosis HLA class II genotyping distinguishes differences of clinical phenotype across ethnic groups. Hum Mol Genet. 2010 Oct;19(20):4100-11.

15 Zissel G, Prasse A, Müller-Quernheim J. Immunologic response of sarcoidosis. Semin Respir Crit Care Med. 2010 Aug;31(4):390-403. 


\section{Case Reports in Ophthalmology}

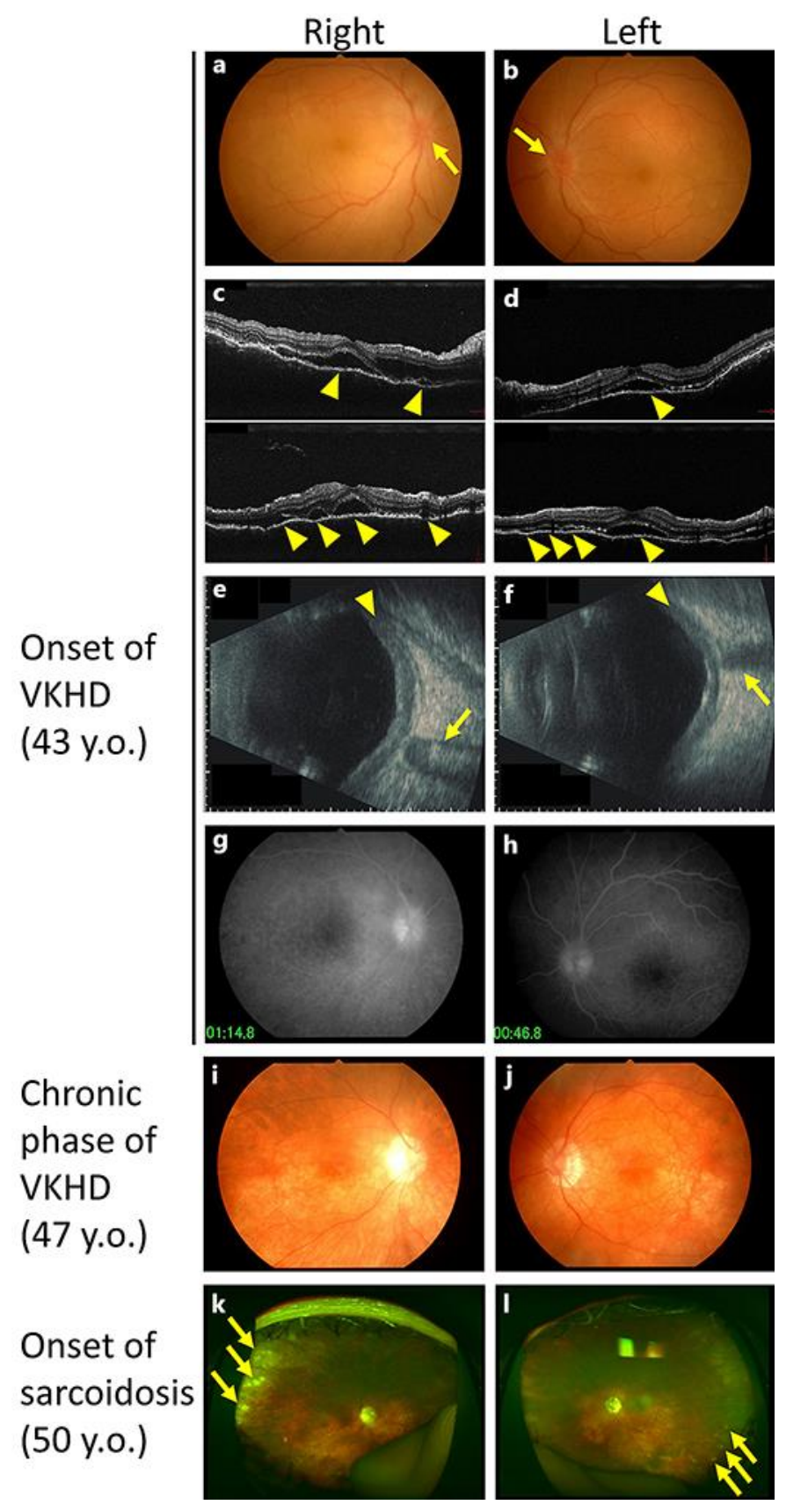

Fig. 1. Ophthalmological examinations in the acute phase of Vogt-Koyanagi-Harada disease (VKHD) at the age of $43(\mathbf{a}-\mathbf{h})$, the chronic phase of VKHD at the age of $47(\mathbf{i}, \mathbf{j})$, and at the onset of sarcoidosis at the age of $50(\mathbf{k}, \mathbf{l})$. Color retinal photographs of the right (a) and left eyes (b) showing swollen and hyperemic optic discs (arrows). Optical coherence tomography of the right (c) and left eyes (d) showing multifocal serous retinal detachments (arrowheads). Ultrasonic B-mode examination of the right (e) and left eyes (f) showing diffuse thickening of the choroid with low to medium reflectivity (arrowheads). The arrows in $\mathbf{e}$ and $\mathbf{f}$ indicate the optic nerves. Fundus fluorescein angiography of the right (g) and left eyes (h) showing optic disc leakage and numerous hyperfluorescent pinpoints of leakage. Color retinal photographs of the right (i) and left eyes (j) showing "sunset glow fundus." Color retinal photographs of the right (k) and left eyes (I) showing Dalen-Fuchs atrophic nodules in the retinal periphery (arrows), which are typical of VKHD. 


\section{Case Reports in Ophthalmology}

\begin{tabular}{l|l}
\hline Case Rep Ophthalmol 2019;10:32-40 \\
\hline DOI: 10.1159/000496384 & $\begin{array}{l}\text { @ 2019 The Author(s). Published by S. Karger AG, Basel } \\
\text { www.karger.com/cop }\end{array}$ \\
\hline
\end{tabular}

Sehara et al.: Neurosarcoidosis Occurring 6 Years after Onset of Vogt-Koyanagi-Harada Disease
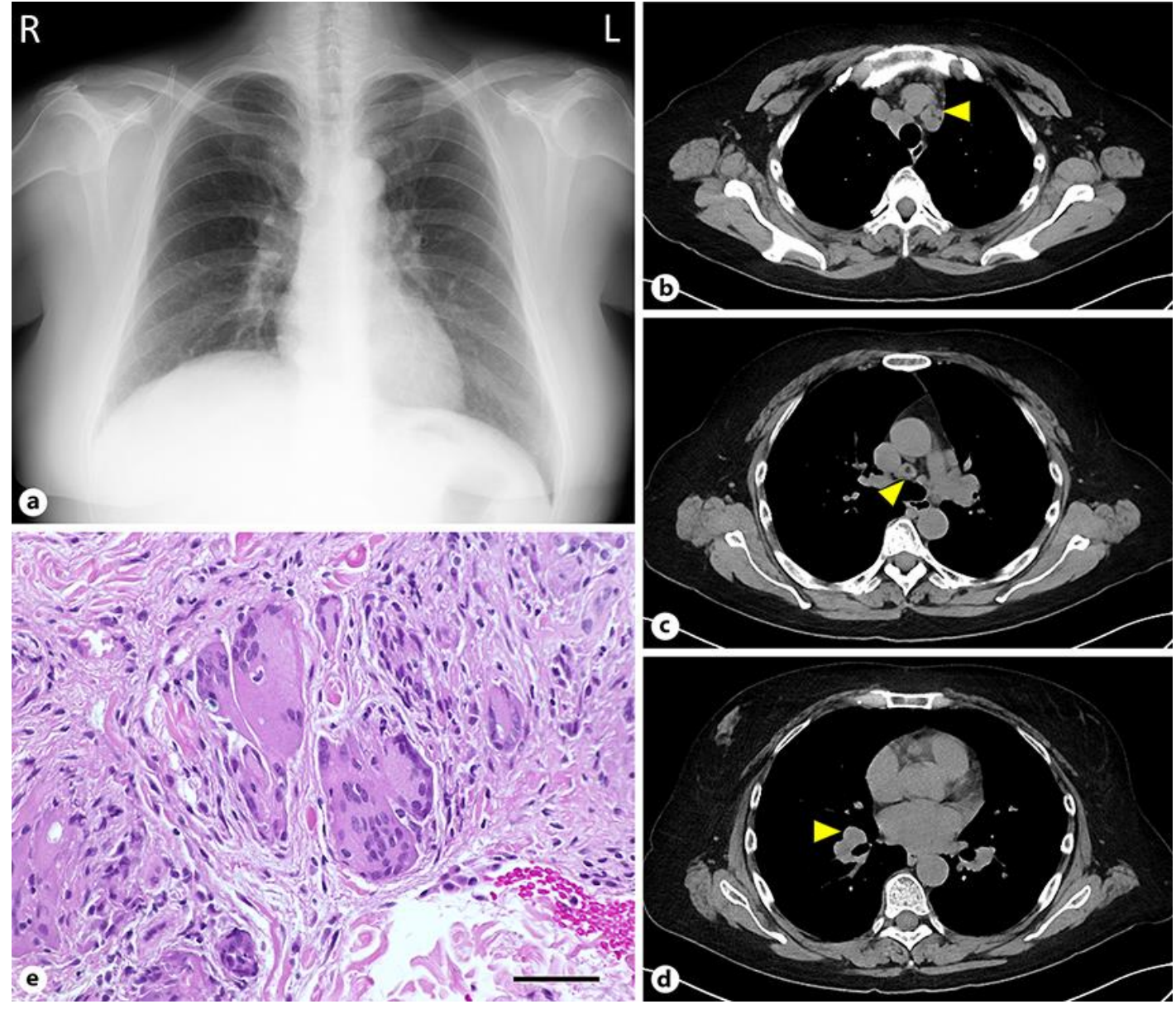

Fig. 2. a Chest X-ray at the age of 50 showing no notable findings, including bilateral hilar lymphadenopathy, which is typical of sarcoidosis. $\mathbf{b}-\mathbf{d}$ Chest CT showing enlargement of some mediastinal lymph nodes (arrowheads). e Histopathology of subcutaneous nodule in the right arm showing multinucleated giant cells. Scale bar $=50 \mu \mathrm{m}$. 

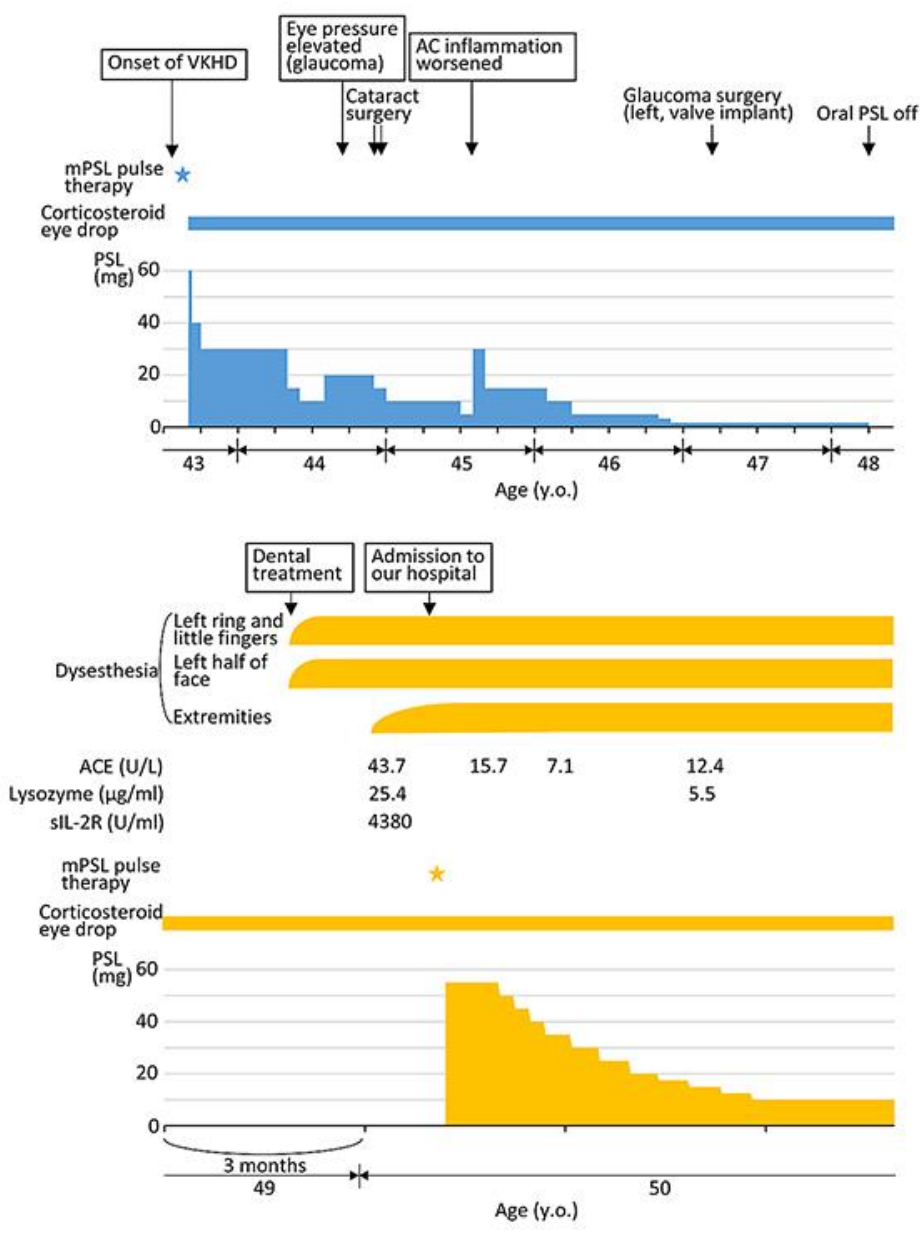

Fig. 3. Clinical course in this case. AC, anterior chamber; ACE, angiotensin-converting enzyme; mPSL, methylprednisolone; PSL, prednisolone; sIL-2R, soluble interleukin-2 receptor; VKHD, Vogt-KoyanagiHarada disease. 